\title{
Successful Use of the Hybrid Assistive Limb for Care Support to Reduce Lumbar Load in a Simulated Patient Transfer
}

\author{
Kousei Miura ${ }^{1,2}$, Hideki Kadone ${ }^{3}$, Tetsuya Abe ${ }^{1}$, Masao Koda ${ }^{1}$, Toru Funayama ${ }^{1}$, Hiroshi Noguchi ${ }^{1}$, \\ Hiroshi Kumagai ${ }^{1}$, Katsuya Nagashima ${ }^{1}$, Kentaro Mataki ${ }^{1}$, Yosuke Shibao ${ }^{1}$, Kosuke Sato ${ }^{1}$, \\ Hiroaki Kawamoto ${ }^{4}$, Yoshiyuki Sankai ${ }^{4}$, Masashi Yamazaki ${ }^{1}$ \\ ${ }^{1}$ Department of Orthopaedic Surgery, Faculty of Medicine, University of Tsukuba, Tsukuba, Japan \\ ${ }^{2}$ Department of Rehabilitation Medicine, Faculty of Medicine, University of Tsukuba Hospital, Tsukuba, Japan \\ ${ }^{3}$ Center for Innovative Medicine and Engineering, University of Tsukuba Hospital, Tsukuba, Japan \\ ${ }^{4}$ Center for Cybernics Research, University of Tsukuba, Tsukuba, Japan
}

Study Design: Prospective experimental study in humans.

Purpose: To determine whether the hybrid assistive limb (HAL) for Care Support can reduce lumbar load during a patient transfer. Overview of Literature: The prevalence of work-related low back pain (LBP) among nurses is high. In particular, transferring patients poses a high risk for LBP due to the large lumbar load. Attempts to reduce the lumbar load are crucial to avoid the risk of LBP. Therefore, we investigated the effects of the HAL for Care Support.

Methods: Nineteen volunteers (16 men, three women) lifted a 60-kg doll from a seated position to a standing position. The first transfer was performed without the HAL for Care Support, and the second was performed with the HAL for Care Support assistive robot. We evaluated transfer performance, the visual analog scale (VAS) score for lumbar fatigue, and electromyogram analyses of the trunk and hip.

Results: Four participants (two men, two women) succeeded with the HAL for Care Support even though they were unable to perform the task without it. The mean lumbar fatigue VAS score for all participants without the HAL for Care Support was $62 \mathrm{~mm}$, while that with it was $43 \mathrm{~mm}$. With lumbar assistance from the HAL for Care Support, subjective lumbar fatigue during the transfer decreased significantly. A power analysis indicated adequate statistical power to detect a difference in the VAS score for lumbar fatigue (0.99). The activity of the left gluteus maximus alone increased significantly during transfers with the HAL for Care Support. No adverse events occurred during use of the HAL for Care Support for transfers.

Conclusions: The HAL for Care Support was able to reduce lumbar load in a simulated patient transfer.

Keywords: Low back pain; Exoskeleton device; Patient transfer; Nurses

\section{Introduction}

Work-related low back pain (LBP) is a serious socio- economic problem because of the large economic costs, including direct costs (medical costs, such as medication and physical therapy) and indirect costs (absences and

Received Apr 12, 2019; Revised Sep 12, 2019; Accepted Oct 10, 2019

Corresponding author: Kousei Miura

Department of Orthopaedic Surgery, Faculty of Medicine, University of Tsukuba, Tennodai, Tsukuba, Ibaraki, 305-8575, Japan

Tel: +81-29-853-3219, Fax: +81-29-853-3162,E-mail: kmiura@tsukuba-seikei.jp 
decreased productivity). Studies have shown that the physical and psychological load at work can cause workrelated LBP. Flexion and rotation of the trunk and lifting are risk factors for LBP [1]. In addition, the prevalence of back pain increases as the number of working hours spent on repeated strenuous physical activities and repeated bending, twisting, or reaching increases [2]. Regarding psychological factors, job satisfaction, monotonous tasks, work relationships, demands, stress, and perceived ability to work are related to future back pain problems [3]. The prevalence of LBP among nurses worldwide is particularly high [4-8]. Among the various tasks that nurses perform, independently transferring patients between beds and chairs poses a high risk for LBP because of the large lumbar load [9]. Thus, attempts to decrease the lumbar load are crucial to avoid the risk of LBP. Previous studies have evaluated the effects of interventions, such as electromechanical lifts and sliding lift sheets, to reduce the lumbar load during patient transfers [10-12].

There are few reports evaluating the lumbar load for nurses using a wearable robot. Therefore, we investigated the hybrid assistive limb (HAL) for Care Support (Cyberdyne Inc., Tsukuba, Japan). The HAL is a novel robot suit that was developed to assist joint motion. It can provide interactive motion according to the wearer's voluntary motor drive. Various types of HALs, such as those for lower limbs, for single joints, and for lumbar support, have been used for musculoskeletal and neurological disorders, including cerebral infarction, spinal cord injury, myelopathy, and osteoarthritis [13-15]. The HAL for Care Support substitutes hip joint motion for trunk motion and assists hip joint extension, which thereby reduces the lumbar load during lifting. It comprises an exoskeletal frame, power units, and lumbar and thigh molds. Tightening the lumbar mold enables trunk motion restraint. The torque assisting the wearer's hip extension is affected by actuators within power units located bilaterally on the wearer's greater trochanters. Nerve and muscle action potentials of the lumbar erector spinae are detected as bioelectrical signals (BES) through electrodes attached to the skin so the HAL for Care Support can sense the wearer's intention to lift something. Moreover, the absolute angle of the trunk is measured by a triaxial accelerometer within the exoskeletal frame. The relative angles of the hip joint are measured by angular sensors within the power units and potentiometers. It is possible for motion supports to coordinate the level and timing of the torque because of these mechanisms of the HAL for Care Support, which comprises a hybrid control system composed of two subsystems: the cybernic voluntary control (CVC) and cybernic autonomous control (CAC) systems [16]. The CVC system performs concerted support that reflects the wearer's voluntary motion thorough BES. The CAC system performs compensatory support against gravity on the wearer's weight to decrease the moment induced by trunk flexion. In addition, the HAL for Care Support can be easily put on without the help of others in a few minutes. The wearer can even run and jump without difficulty. Thus far, the HAL for Care Support has been reported to reduce lumbar loads and improve task performance in repetitive lifting and snow-shoveling movements $[17,18]$. However, our search of the literature revealed no studies evaluating lumbar load during patient transfer movements using the HAL for Care Support. The aim of the present study was to determine whether the HAL for Care Support could reduce the lumbar load during a patient transfer.

\section{Materials and Methods}

\section{Participants}

Inclusion criteria for this study were volunteer participants aged 20-45 years who were healthy, injury-free, and suitable for the HAL for Care Support. Volunteers with heart and respiratory diseases undergoing medical treatment and with LBP were excluded. Nineteen volunteers (16 men, three women) participated in this study. The mean age of the volunteers was 31 years (range, 27-45 years); the mean height was $172 \pm 8.9 \mathrm{~cm}$ (range, 155-184 $\mathrm{cm}$ ), and the mean weight was $66 \pm 11 \mathrm{~kg}$ (range, 47-85 $\mathrm{kg}$ ). The mean finger-to-floor distance, which demonstrates the flexibility of the trunk, was $4.0 \pm 7.4 \mathrm{~cm}$ (range, -11 to $16 \mathrm{~cm}$ ). The mean back-muscle strength (as measured by TKK5402; Takei Scientific Instruments Co., Niigata, Japan) was $110 \pm 59 \mathrm{~kg}$ (range, 51-170 kg). Informed consent was obtained from all participants in this study. The study was performed in accordance with the Declaration of Helsinki and within the appropriate ethical framework (IRB approval no., H27-50).

\section{Patient transfer protocol}

The subject for transfer was a doll weighing $60 \mathrm{~kg}$, which simulated a patient with complete cervical spinal cord in- 

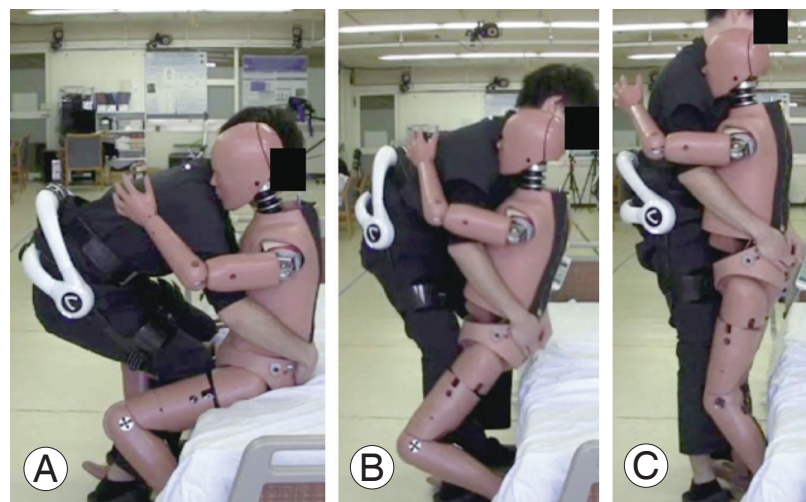

Fig. 1. (A-C) A study participant performing a simulated patient transfer with the hybrid assistive limb for Care Support (Cyberdyne Inc., Tsukuba, Japan). The subject for transfer was a $60-\mathrm{kg}$ doll.

jury. Initially, the doll was in a seated position at bedside. Participants adopted a half-seated position to initiate the transfer. The doll's arms were placed on the participant's shoulders. Participants held the doll in their arms and lifted it until the doll was in a standing position (Fig. 1). The first transfer was performed without the HAL for Care Support. After a resting period of at least 5 minutes, the second transfer was conducted with the participant wearing the HAL for Care Support suit.

\section{Outcome measures and statistical analysis}

We evaluated the transfer performance and lumbar load and determined whether participants could lift the doll to a standing position. A visual analog scale (VAS) score for lumbar fatigue (maximum score, $100 \mathrm{~mm}$ ) was determined after each trial to evaluate the lumbar load. Furthermore, wireless surface electromyogram analyses (Trigno Lab System; Delsys Inc., Natick, MA, USA) with six electromyogram sensors bilaterally attached to the latissimus dorsi, lumbar elector spinae, and gluteus maximus were conducted to evaluate muscle activity during the transfer. Participants were asked to report adverse events during the transfer for the safety evaluation of the HAL for Care Support in this transfer protocol. A Wilcoxon signed-rank test was used to compare variables when transferring patients with and without the HAL for Care Support. All statistical analyses were performed using the JMP software package ver. 14.0.0 (SAS Institute Inc., Cary, NC, USA). For all comparisons, $p<0.05$ was considered significant.

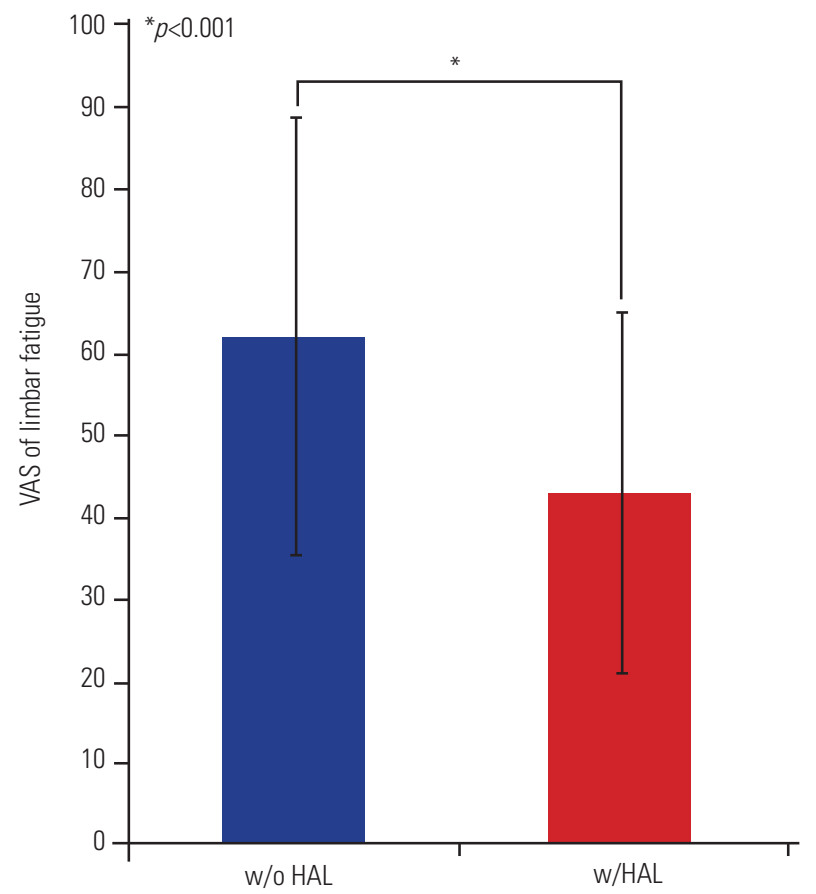

Fig. 2. Mean VAS of lumbar fatigue. W/o HAL, transfer without the HAL for Care Support; W/ HAL, transfer with the HAL for Care Support. VAS, visual analog scale; HAL, hybrid assistive limb. " $p<0.001$.

\section{Results}

The transfer was completely accomplished by 14 of the male volunteers regardless of whether they were equipped with the HAL for Care Support. Four participants (two men, two women) succeeded with the transfer using the HAL for Care Support even though they could not succeed without it. Only one female volunteer could not accomplish the transfer task regardless of whether she used the HAL for Care Support. The mean VAS score of lumbar fatigue for all participants without the HAL for Care Support was $62 \pm 26 \mathrm{~mm}$ (range, 7-91 mm), while that with the HAL for Care Support was $43 \pm 22 \mathrm{~mm}$ (range, 0-79 mm) (Fig. 2). With lumbar support from the HAL for Care Support, subjective lumbar fatigue during the transfer decreased significantly. Muscle activity could not be evaluated in 10 participants because of noise in the electromyograms. We analyzed the muscle activity of the eight participants whose electromyograms could be evaluated. Only the activity of the left gluteus maximus increased significantly during the transfer with the HAL for Care Support compared with the transfer without the HAL for Care Support. The activity of the other muscles did not significantly change. There were no adverse events when using the HAL for Care Support for transferring the doll. 


\section{Discussion}

It has been recognized that many nurses have musculoskeletal disorders because of their heavy workload. There are many studies on the particularly high prevalence of LBP among nurses worldwide [4-8]. Several systematic reviews have summarized the prevalence of LBP. Hignett [19] reported that the annual prevalence of LBP ranged from $40 \%$ to $50 \%$, and the lifetime prevalence ranged from $35 \%$ to $80 \%$. Azizpour et al. [20] showed that the prevalence of LBP during working life and during a year was estimated at $63 \%$ and $61.2 \%$, respectively. Lorusso et al. [21] reported that the 12-month prevalence of LBP ranged from $33 \%$ to $86 \%$. Of the nursing tasks causing LBP, manually transferring patients between a bed and a chair is significantly associated with an increased risk of back pain $[9,22]$. Moreover, the spinal biomechanical load during a patient transfer exceeds tolerance limits, and patient transfer is associated with a risk of low back disorder [23]. To avoid the risk of LBP in nurses, training or education, no-lift policies and several devices to assist patient transfers, including battery-powered lifts, sliding boards, and ceilingbased patient lifts, have been attempted [10-12]. However, these interventions have not been established to prevent LBP because there is no strong evidence for their efficacy according to systematic reviews [24-26].

In the present study, we evaluated the safety of the HAL for Care Support and investigated whether it could reduce lumbar load and improve performance during the transfer of a doll that simulated a patient with complete cervical spinal cord injury. No adverse events occurred while participants were using the HAL for Care Support for this task. Without the HAL for Care Support, five of the 18 participants failed in the simulated patient transfer.

However, with the HAL for Care Support, four of these five participants succeeded, which indicates the HAL for Care Support could improve transfer performance. The HAL for Care Support significantly reduced subjective lumbar fatigue. A power analysis indicated adequate statistical power to determine differences in the VAS score of lumbar fatigue (0.99). Detecting muscle activity was a problem during the analysis because the electromyograms of approximately half of the participants could not be evaluated due to noise. In the eight participants whose electromyograms could be evaluated, only the activity of the left gluteus maximus increased significantly during the transfer with the HAL for Care Support compared with the transfer without the HAL for Care Support. The HAL for Care Support replaces trunk motion with hip joint motion and assists extension. The HAL for Care Support might increase the muscle activity of the gluteus maximus by assisting the hip joint motion so that participants can perform the simulated patient transfer movements more easily.

There are some limitations to the present study. Only the vertical movement of a patient was simulated and evaluated in this study. In addition to a vertical load, actual patient transfers require a trunk rotational load. Electromyogram analysis linked to motion analysis, such as that in the joint angles of the trunk and hip, remains to be investigated for more precise biomechanical evaluation. Additionally, initial implementation costs and maintenance costs are required to use the HAL for Care Support. Thus, its cost-effectiveness compared with other interventions should be considered. Further studies with highquality study designs are needed to confirm that the HAL for Care Support can reduce lumbar load.

\section{Conclusions}

The HAL for Care Support significantly reduced subjective lumbar fatigue in a simulated patient transfer. Use of the HAL for Care Support may reduce the occurrence of low back disorders.

\section{Conflict of Interest}

A commercial party having a direct financial interest in the results of the research supporting this article has conferred or will confer a financial benefit on one or more of the authors. Yoshiyuki Sankai is a professor of University of Tsukuba, a founder, a shareholder, and the chief executive officer of university venture company "CYBERDYNE Inc.", Ibaraki, Japan. Hiroaki Kawamoto is an associate professor of University of Tsukuba, a co-founder, a shareholder, and an outside director of university venture company CYBERDYNE. Conflict of interest is managed by University of Tsukuba according to national university rules and guidelines and by board of directors of CYBERDYNE. The patent royalty is paid to University of Tsukuba from CYBERDYNE and a part of the royalty is paid to Yoshiyuki Sankai from University of Tsukuba according to national university rules. CYBERDYNE is a R\&D company and the manufacturer of the HAL. The present 
study was proposed by the authors. CYBERDYNE was not directly involved in the study design; collection, analysis, or interpretation of data; writing the report; or the decision to submit the paper for publication. No commercial party having a direct financial interest in the results of the research supporting this article has or will confer a benefit on the authors or on any organization with which the other authors are associated (Kousei Miura, Hideki Kadone, Tetsuya Abe, Masao Koda, Toru Funayama, Hiroshi Noguchi, Hiroshi Kumagai, Katsuya Nagashima, Kentaro Mataki, Yosuke Shibao, Kosuke Sato, and Masashi Yamazaki). No potential conflict of interest relevant to this article was reported.

\section{Acknowledgments}

We thank Mayuko Sakamaki and Yumiko Ito, Center for Innovative Medicine and Engineering (CIME), University of Tsukuba Hospital, for their excellent technical assistance. This work was supported by an Industrial Disease Clinical Research Grant from the Ministry of Health Labour and Welfare, Japan (grant no., 160401-01).

\section{Author contributions}

All authors read and approved the final manuscript.

\section{References}

1. Hoogendoorn WE, Bongers PM, de Vet HC, Ariens GA, van Mechelen W, Bouter LM. High physical work load and low job satisfaction increase the risk of sickness absence due to low back pain: results of a prospective cohort study. Occup Environ Med 2002;59:323-8.

2. Guo HR. Working hours spent on repeated activities and prevalence of back pain. Occup Environ Med 2002;59:680-8.

3. Linton SJ. Occupational psychological factors increase the risk for back pain: a systematic review. J Occup Rehabil 2001;11:53-66.

4. Trinkoff AM, Lipscomb JA, Geiger-Brown J, Brady B. Musculoskeletal problems of the neck, shoulder, and back and functional consequences in nurses. Am J Ind Med 2002;41:170-8.

5. Smith DR, Kondo N, Tanaka E, Tanaka H, Hirasawa K, Yamagata Z. Musculoskeletal disorders among hospital nurses in rural Japan. Rural Remote Health 2003;3:241.

6. Shieh SH, Sung FC, Su CH, Tsai Y, Hsieh VC. Increased low back pain risk in nurses with high workload for patient care: a questionnaire survey. Taiwan J Obstet Gynecol 2016;55:525-9.

7. Hofmann F, Stossel U, Michaelis M, Nubling M, Siegel A. Low back pain and lumbago-sciatica in nurses and a reference group of clerks: results of a comparative prevalence study in Germany. Int Arch Occup Environ Health 2002;75:484-90.

8. Samaei SE, Mostafaee M, Jafarpoor H, Hosseinabadi MB. Effects of patient-handling and individual factors on the prevalence of low back pain among nursing personnel. Work 2017;56:551-61.

9. Smedley J, Egger P, Cooper C, Coggon D. Manual handling activities and risk of low back pain in nurses. Occup Environ Med 1995;52:160-3.

10. Zhuang Z, Stobbe TJ, Hsiao H, Collins JW, Hobbs GR. Biomechanical evaluation of assistive devices for transferring residents. Appl Ergon 1999;30:285-94.

11. Peterson EL, McGlothlin JD, Blue CL. The development of an ergonomics training program to identify, evaluate, and control musculoskeletal disorders among nursing assistants at a state-run veterans' home. J Occup Environ Hyg 2004;1:D10-6.

12. Humphreys SL. Obesity in patients and nurses increases the nurse's risk of injury lifting patients. Bariatr Nurs Surg Patient Care 2007;2:3-6.

13. Kubota S, Nakata Y, Eguchi K, et al. Feasibility of rehabilitation training with a newly developed wearable robot for patients with limited mobility. Arch Phys Med Rehabil 2013;94:1080-7.

14. Yoshikawa K, Mutsuzaki H, Sano A, et al. Training with hybrid assistive limb for walking function after total knee arthroplasty. J Orthop Surg Res 2018;13:163.

15. Kubota S, Kadone H, Shimizu Y, et al. Robotic rehabilitation training with a newly developed upper limb single-joint Hybrid Assistive Limb (HAL-SJ) for elbow flexor reconstruction after brachial plexus injury: a report of two cases. J Orthop Surg (Hong Kong) 2018;26:2309499018777887.

16. Hara H, Sankai Y. Development of HAL for lumbar support. Proceedings of the SCIS and ISIS 2010: Joint 5th International Conference on Soft Computing and Intelligent Systems and 11th International 
Symposium on Advanced Intelligent Systems; 2010 Dec 8-12; Okayama, Japan. Okayama: Committee of SCIS-ISIS; 2010. p. 416-21.

17. Miura K, Kadone H, Koda M, et al. The hybrid assisted limb (HAL) for Care Support, a motion assisting robot providing exoskeletal lumbar support, can potentially reduce lumbar load in repetitive snowshoveling movements. J Clin Neurosci 2018;49:83-6.

18. Miura K, Kadone H, Koda M, et al. The hybrid assistive limb (HAL) for Care Support successfully reduced lumbar load in repetitive lifting movements. J Clin Neurosci 2018;53:276-9.

19. Hignett S. Work-related back pain in nurses. J Adv Nurs 1996;23:1238-46.

20. Azizpour Y, Delpisheh A, Montazeri Z, Sayehmiri K. Prevalence of low back pain in Iranian nurses: a systematic review and meta-analysis. BMC Nurs 2017;16:50.

21. Lorusso A, Bruno S, L'Abbate N. A review of low back pain and musculoskeletal disorders among Italian nursing personnel. Ind Health 2007;45:637-44.
22. Choi SD, Brings K. Work-related musculoskeletal risks associated with nurses and nursing assistants handling overweight and obese patients: a literature review. Work 2015;53:439-48.

23. Marras WS, Davis KG, Kirking BC, Bertsche PK. A comprehensive analysis of low-back disorder risk and spinal loading during the transferring and repositioning of patients using different techniques. Ergonomics 1999;42:904-26.

24. Hegewald J, Berge W, Heinrich P, et al. Do technical aids for patient handling prevent musculoskeletal complaints in health care workers?: a systematic review of intervention studies. Int J Environ Res Public Health 2018;15:E476.

25. Richardson A, McNoe B, Derrett S, Harcombe H. Interventions to prevent and reduce the impact of musculoskeletal injuries among nurses: a systematic review. Int J Nurs Stud 2018;82:58-67.

26. Van Hoof W, O'Sullivan K, O’Keeffe M, Verschueren S, O'Sullivan P, Dankaerts W. The efficacy of interventions for low back pain in nurses: a systematic review. Int J Nurs Stud 2018;77:222-31. 